\title{
Uma Metaheurística GRASP/VNS para uma Solução Aproximada do Problema de Recobrimento de Rotas
}

L.C.S. MOTTA ${ }^{1}$, Departamento de Ciência da Computação, Universidade Federal Fluminense, Rua Passo da Pátria, 156, 24210-240 Niterói, RJ, Brasil

L.S. OCHI ${ }^{2}$, Departamento de Ciência da Computação, Universidade Federal Fluminense, Rua Capitão Zeferino, 56/808 - Icaraí 24220-230 Niterói, RJ, Brasil

C.A. MARTINHON ${ }^{3}$, Departamento de Ciência da Computação, Universidade Federal Fluminense, Rua Passo da Pátria, 156, 24210-240 Niterói, RJ, Brasil.

\begin{abstract}
Resumo. O Problema de Recobrimento de Rotas (PRR) é um problema de sequenciamento de tarefas definido sob um grafo $G=(V \cup W, E)$, onde $W$ é o conjunto de vértices que devem ser cobertos. O problema consiste em determinar uma rota ou um ciclo Hamiltoniano de comprimento mínimo sob um subconjunto de $V$, de modo que todo vértice de $W$ esteja a uma distância não negativa $d$ de algum vértice da rota. Sendo uma generalização do Problema do Caixeiro Viajante (PCV), o PRR é considerado NP-difícil. Este trabalho apresenta: uma nova formulação matemática baseada em variáveis de fluxo, regras para a redução do grafo associado e uma metaheurística original para solucionar aproximadamente o PRR.
\end{abstract}

\section{Introdução}

O impacto das novas tecnologias em computação tem demostrado uma necessidade cada vez maior em resolver problemas até então desconsiderados. Diante das diversas mudanças no tratamento de problemas difíceis de otimização, que englobam os problemas do mundo real, os métodos clássicos de otimização têm encontrado grandes dificuldades para obter uma solução ótima (ótimo global), mesmo quando estes métodos possuem teoricamente a garantia de atingí-la. A grande quantidade de problemas de otimização de elevada complexidade encontrados em diferentes áreas, tem provocado a necessidade de se pesquisar cada vez mais o desenvolvimento de novos métodos eficientes não só do ponto de vista teórico, como também do ponto de vista operacional. Esses métodos são usualmente o resultado da adaptação de

\footnotetext{
${ }^{1}$ luciene.motta@pgcc.uff.br

2 satoru@dcc.ic.uff.br

3 mart@dcc.ic.uff.br
} 
idéias de uma grande variedade de áreas, esperando que se produza procedimentos eficientes e hábeis para lidar com problemas cada vez mais complexos e dinâmicos. Um destes procedimentos, chamado de metaheurística possue como principal característica a existência de ferramentas que possibilitam evitar uma parada prematura em ótimos locais ainda distantes de uma solução ótima do problema e a flexibilidade, responsável por uma melhor adaptação ao espaço de busca. São propostos neste trabalho: uma nova formulação matemática para o Problema de Recobrimento de Rotas, um estudo de regras para a redução do grafo a ele associado, assim como um novo algoritmo aproximado.

\section{O Problema de Recobrimento de Rotas}

Inicialmente definido por John Current [1], o Problema de Recobrimento de Rotas (PRR), conhecido na literatura por Covering Tour Problem (CTP), é uma extensão do Problema do Caixeiro Viajante (Traveling Salesman Problem - TSP) e pode ser definido da seguinte maneira:

Seja $G=(V \cup W, E)$ um grafo não direcionado, onde $V \cup W=\{1, \ldots, n\}$ denota o conjunto de vértices, e $E=\{(i, j) \mid i, j \in V \cup W, i<j\}$ o conjunto de arestas. O vértice $(s=1)$ representa o vértice origem; $V$ o conjunto dos vértices que podem ser visitados; $T \subseteq V$ o conjunto dos vértices que devem ser visitados $(s \in T)$, e $W$ o conjunto dos vértices que devem ser cobertos. A matriz de distância $C=\left(c_{i j}\right)$, definida sob o conjunto de arestas E, utiliza a métrica Euclideana.

O PRR consiste em determinar uma rota ou um ciclo Hamiltoniano de comprimento mínimo sob um subconjunto de $V$. Esta rota deve conter todos os vértices de $T$ e deve cobrir cada vértice de $W$. Um vértice $i \in W$ é considerado coberto se distar no máximo $d$ de algum vértice da rota, onde $d$ é um dado de entrada.

O PRR é considerado NP-difícil uma vez que se reduz ao Problema do Caixeiro Viajante quando $d=0$ e todos os vértices de $V$ coincidem com os vértices de $W$, ou seja, $V=W$.

Apesar de possuir diversas aplicações, o PRR não tem recebido muita atenção na literatura afim. De nosso conhecimento, somente poucas heurísticas e dois métodos exatos foram apresentados para o problema. Gendreau et al. [3] combinam uma heurística para o PCV com outra para o Problema de Recobrimento de Vértices (Set Covering Problem). Maniezzo et al. [4] apresentam três versões da metaheurística Scatter Search para o PRR.

\section{Procedimentos Propostos}

Em Otimização Combinatória, muitos problemas são classificados como NP-difíceis, dentre eles as diversas generalizações do PCV. Embora atualmente existam eficientes técnicas exatas para a solução destes problemas, reconhecemos que a sua complexidade dificulta ou até inviabiliza a solução de instâncias de elevadas dimensões, como as associadas a problemas reais. Uma alternativa tem sido o uso de técnicas aproximadas ou heurísticas no sentido de obter soluções de boa qualidade em um tempo 
computacional suportável. Contudo, o simples uso destas técnicas pode não ser suficiente para atingir objetivos que conjugam qualidade com tempos computacionais aceitáveis. Este trabalho propõe um conjunto de testes de redução para o PRR a ser incorporado tanto em métodos exatos quanto aproximados, cujo objetivo é manter a qualidade da solução final utilizando um tempo computacional reduzido. Além dos testes de redução, também é proposta uma descrição mais genérica para o PRR e uma nova formulação matemática introduzindo variáveis de fluxo. Adicionalmente apresentamos um algoritmo metaheurístico que compartilha características de duas metaheurísticas conhecidas como GRASP (Greedy Randomized Adaptive Search Procedures) [2] e VNS (Variable Neigborhood Search) [5].

\section{Formulação Proposta}

Este trabalho define uma generalização PRR, denominado Problema de Recobrimento de Rotas Genérico (PRRG). O PRRG consiste em determinar uma rota de comprimento mínimo sob um subconjunto de vértices de $W$ além de um subconjunto de vértices de $V$. Desta forma, permitimos que os vértices de $W$ também possam ser utilizados para seu próprio recobrimento, como também para cobrir outros vértices deste conjunto numa solução do PRRG.

Problema de Recobrimento de Rotas também pode ser formulado como o Problema do Caixeiro Viajante Generalizado (PCVG) [6], o que torna possível particionar os vértices do PRRG em um conjunto de grupamentos para o problema. Considerando o PRRG, definimos os grupamentos do seguinte modo:

$$
\begin{array}{ll}
C_{i}=\left\{j \in V \cup W \mid c_{i j} \leq d\right\}, & \forall i \in W, \\
C_{t}=\{t\}, & \forall t \in T .
\end{array}
$$

A Figura 1 ilustra uma instância para o PRR com 29 vértices, tendo os seus respectivos grupamentos ilustrados pelas circunferências.

De acordo com a literatura, o PRR foi primeiramente formulado por Current [1]. Mais tarde, Gendreau et al. [3] e Maniezzo et al. [4] apresentaram novas formulações para o problema. A maioria das formulações utilizam restrições tradicionais do PCV para evitar a formação de subciclos desconexos da origem. Nesta seção, é proposta uma nova formulação para o PRRG introduzindo variáveis de fluxo. Na formulação aqui proposta o número de restrições é polinomial no tamanho do problema, ao contrário da maioria das formulações propostas, que exigem um número exponencial de desigualdades associadas às formulações do PRR. A idéia básica da formulação aqui proposta, é associar uma variável de fluxo a cada aresta $(i, j) \in E$. Seja $y_{k}$, para $k \in V$, uma variável binária igual a 1 se e somente se, o vértice $k$ estiver na rota e zero, caso contrário. Se $k \in T$, então $y_{k}$ é necessariamente igual a 1 . Seja $x_{i j}$, para todo $i, j \in V \operatorname{com} i \neq j$, uma variável binária que é igual a 1 se e somente se, a aresta $(i, j)$ pertence à rota e zero, caso contrário. Também são definidos coeficientes binários $\delta_{l k}$ iguais a 1 se e, somente se, $l \in W$ puder ser coberto por $k \in V$ (isto é, se $c_{l k} \leq d$ e $S_{l}=\left\{k \in V \mid \delta_{l k}=1\right\}$ ) e zero, caso contrário. A variável de fluxo associada a cada $(i, j) \in E$, com $i \neq j$, é definida como uma variável inteira não negativa, $z_{i j}$, que representa a quantidade de fluxo escoado no 


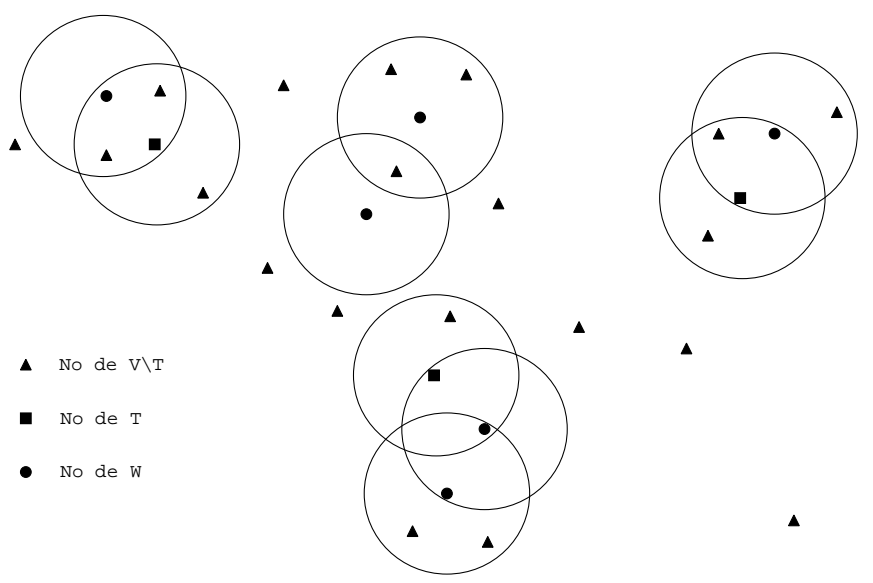

Figura 1: Formação dos grupamentos dos nós do PRRG

$\operatorname{arco}(i, j)$. De posse destas considerações, a formulação apresentada é descrita da seguinte forma:

$$
\begin{gathered}
(P R R G) \text { minimizar } \sum_{i<j \mid i, j \in V} c_{i j} x_{i j}, \quad s a: \\
\sum_{k \in S_{l}} y_{k} \geq 1 \quad(\forall l \in W), \\
\sum_{i<k} x_{i k}+\sum_{k<j} x_{k j}=2 y_{k} \quad(\forall k \in V \cup W), \\
\sum_{j \in V \cup W} z_{k j}=\sum_{i \in V \cup W} z_{i k}+y_{k} \quad(\forall k \in V \cup W-\{s\}), \\
\sum_{j \in V \cup W} z_{s j}=1 \quad \sum_{j \in V \cup W} z_{j s}=\sum_{j \in V \cup W} y_{j} \\
x_{i j} \leq z_{i j} \quad(\forall i, \forall j \in V \cup W) ; \\
x_{i j} \geq\left(z_{i j}\right) /(|V|+|W|+1) \quad(\forall i, \forall j \in V \cup W), \\
y_{k}=1 \quad(\forall k \in T), \\
y_{k} \in\{0,1\} \quad x_{i j} \in\{0,1\} \quad(\forall i, \forall j \in V \cup W), \\
(\forall k \in V \backslash T) \quad(\forall i, \forall j \in V \cup W) .
\end{gathered}
$$

Nesta formulação, definidas pelas restrições (3.3)-(3.12), as restrições (3.4) asseguram que cada vértice de $W$ é coberto por pelo menos um vértice da rota. As restrições (3.5) impõe que todo vértice visitado tenha grau 2. As restrições (3.6) e (3.7) asseguram a conectividade da rota. As restrições (3.8) e (3.9), asseguram que 
a rota gerada a partir da variável de fluxo $\left(z_{i j}\right)$ e pela variável de decisão $\left(x_{i j}\right)$ são coincidentes onde $|V|$ denota a cardinalidade do conjunto V. As restrições (3.10) asseguram que cada vértice de $T$ necessariamente faz parte da rota. Finalmente, as restrições (3.11) e (3.12) representam as condições de integralidade do problema.

\section{Testes de Redução para o PRRG}

As dimensões elevadas dos problemas de otimização combinatória, quando associadas à aplicações reais se caracterizam pela grande quantidade de soluções no seu espaço de busca. Pela limitação do tempo computacional disponível e pela complexidade de determinadas técnicas de busca muitas heurísticas acabam explorando apenas um número reduzido de ótimos locais, comprometendo, com isso em alguns casos, a qualidade da solução final obtida. Um dos recursos que pode ser implementado para superar este obstáculo é a utilização de técnicas de redução do espaço de busca, cujo objetivo principal é a eliminação de informações que são consideradas irrelevantes ao estudo do problema e acabam por consumir mais tempo de execução nos algoritmos propostos para solucioná-lo. Os testes apresentados em [3] e [4], para a simplificação do espaço de busca de uma instância do PRR, não funcionam para a versão generalizada (PRRG) que está sendo utilizada neste trabalho. Desta forma, é proposto novos testes de redução para o PRRG. Seja $C o b=\left(c o b_{i j}\right)$ uma matriz $|W| \times|V \cup W|(0-1)$, onde $c o b_{i j}=1$, para $i \in W, \mathrm{j} \in V \cup W$ se e somente se $c_{i j} \leq d$. Os conjuntos $W$ e $V$ podem ser reduzidos utilizando uma única vez as seguintes regras:

- Transformar cada vértice $i \in W$, tal que $c o b_{i k}=1$, com $k \in T$, em um vértice $i \in V \backslash T$;

- Remover todo vértice $i \in V \backslash T$, tal que $\operatorname{cob}_{j i}=0, \forall j \in W$.

Considerando o grafo associado ao PRRG apresentado na Figura 1, a aplicação da primeira regra do teste de redução é ilustrada na Figura 2.

A Figura 3 ilustra as alterações provocadas no grafo ao final da segunda regra do teste.

O grafo reduzido após o teste de redução é apresentado na figura 4.

\section{Metaheurística GRASP/VNS para o PRRG}

Nesta seção, é proposto um algoritmo metaheurístico para solucionar aproximadamente o PRRG, reunindo conceitos de GRASP (Greedy Randomized Adaptive Search Procedures) [2] e VNS (Variable Neighborhood Search Procedures) [5].

A metaheurística GRASP é um método iterativo, randômico e adaptativo onde cada iteração possui duas fases: uma fase de construção, seguida de uma fase de melhorias, cujo objetivo é encontrar uma solução ótima local. A fase de construção é também iterativa por construir uma solução elemento a elemento e é gulosa por selecionar aleatoriamente cada elemento da solução a partir de um subconjunto que relaciona os melhores candidatos. Uma importante característica desta fase 


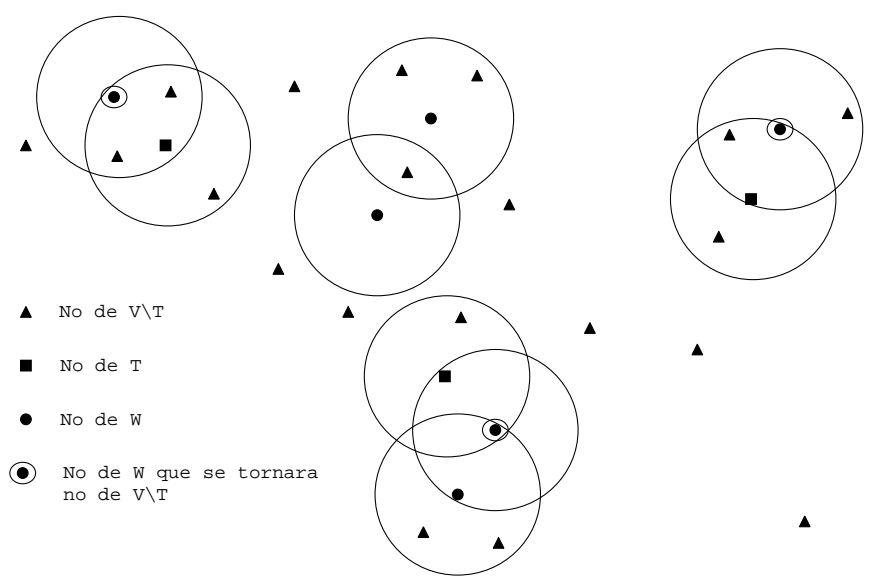

Figura 2: Ilustração dos nós do conjunto $W$ transformados em nós optativos de $(V \backslash T)$

é a adaptação da solução, uma vez que os benefícios associados a cada elemento são levados de uma iteração para outra, refletindo as mudanças ocasionadas pela seleção prévia de outros elementos. A fase de melhorias consiste tipicamente em um procedimento de busca local, já que a solução gerada na fase de construção do GRASP pode não ser um ótimo local.

No algoritmo proposto, a primeira fase realiza o sequenciamento do conjunto de todos os grupamentos do grafo e a segunda fase, encontra a melhor rota a partir dos grupamentos sequenciados na fase anterior. A primeira fase se inicia com a escolha aleatória de um grupamento . Este será o primeiro grupamento da seqüência. Os demais serão escolhidos iterativamente a partir de uma lista de candidatos que estará diretamente relacionada ao grupamento sequenciado mais recentemente. Se o tamanho desta lista for elevado, partimos para a construção de uma lista de candidatos restrita (LCR) a cada passo do algoritmo de construção. Quando não mais existirem grupamentos a serem sequenciados, o algoritmo iniciará a segunda fase. A segunda fase tem a finalidade de definir que elemento (somente um) de cada grupamento pertencerá à rota. Adaptamos a idéia de Maniezzo et al. [4] para solucionar este problema. Obtida uma permutação dos grupamentos na primeira fase do algoritmo, a solução viável associada a esta sequencia será o caminho mínimo desta permutação. Como os grupamentos não são disjuntos, soluções inviáveis podem ser obtidas no final da segunda fase. Sendo assim, fez-se necessário a inclusão de um procedimento para a eliminação de subciclos. O critério adotado para este procedimento é eliminar os ciclos que apresentarem o maior acréscimo na rota.

A busca local é a fase mais importante do processo e é também a mais dispendiosa computacionalmente. É nesta fase que vários ótimos locais são investigados com o objetivo de se tentar atingir um ótimo global. No algoritmo de busca local proposto, utiliza-se um conjunto de trocas simultâneas entre posições de dois ou mais grupamentos da atual permutação. É nesta fase que as noções de VNS são 


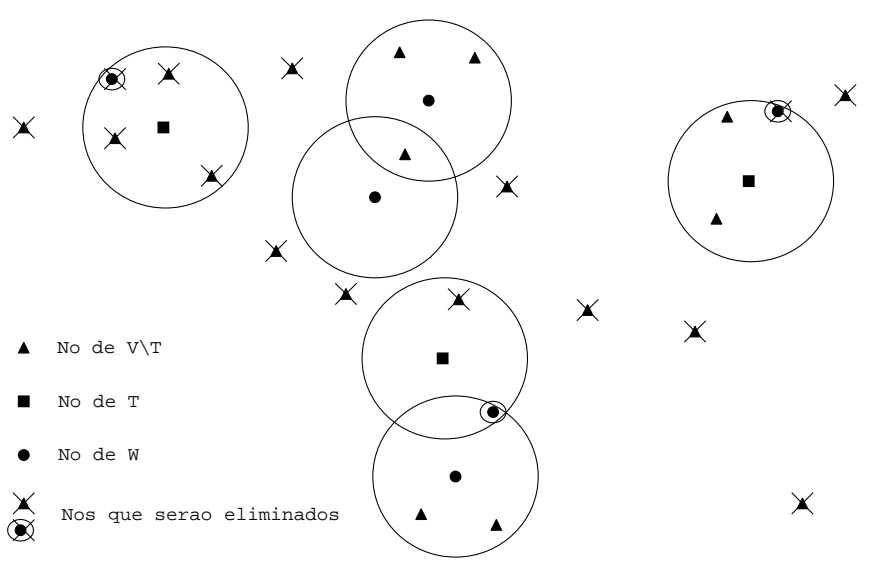

Figura 3: Visualização da eliminação dos nós provocada pela segunda regra do teste de redução

introduzidas. As vizinhanças de uma solução são definidas do seguinte modo: na primeira vizinhança, um par de grupamentos é permutado. Na segunda, dois pares são permutados simultaneamente e assim sucessivamente, na k-ésima vizinhança, $\mathrm{k}$ pares são permutados simultaneamente.

\section{Resultados Computacionais}

As regras de redução têm como objetivo, reduzir o domínio do conjunto de soluções viáveis de um problema.

Para avaliar o desempenho das regras propostas, desenvolvemos um novo algoritmo metaheurístico baseado em conceitos de GRASP e VNS. A Tabela 1 ilustra os resultados médios para instâncias de tamanho de 50 a 300 vértices. As colunas 2 e 3 mostram respectivamente: $|V \cup W|,|T|,|W|,|V \backslash T|$ do grafo original (coluna 2) e grafo reduzido (coluna 3). As colunas 4 e 5 ilustram os resultados médios obtidos pelo método GRASP/VNS sem o uso das regras (coluna 4) e com o uso das regras (coluna 5). Finalmente, a coluna 6 apresenta o percentual médio de melhora na solução obtido pelo algoritmo usando as regras propostas. Em relação ao desempenho médio citado, para cada instância (cada linha da Tabela 1), com uma determinada dimensão do Grafo e dos conjuntos: T, W e V-T, foram criadas aleatóriamente 10 (dez) problemas testes e os resultados de cada linha da Tabela 1, mostram os resultados médios destes 10 problemas testes (colunas 4, 5 e 6).

\section{Conclusões}

Este trabalho apresenta uma generalização do PRR aqui denominado PRRG. Com o objetivo de permitir um uso mais amplo tanto de métodos exatos como apro- 

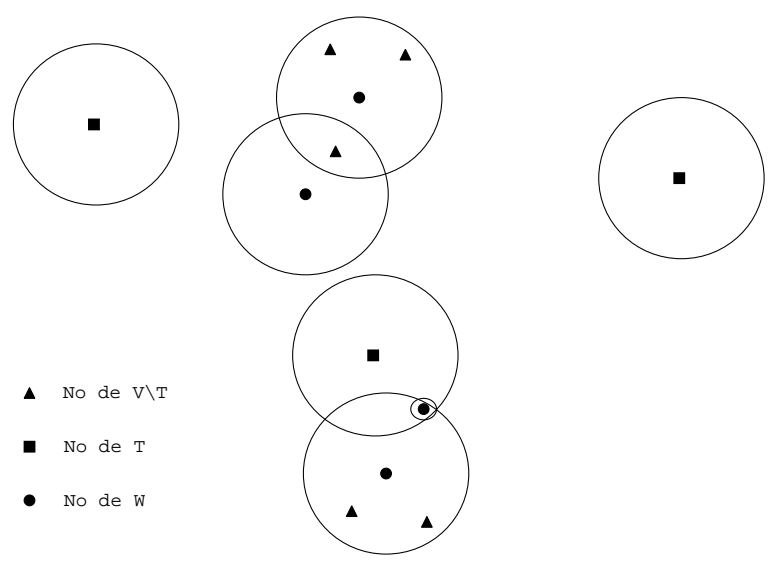

Figura 4: Grafo reduzido após a utilização do teste de redução proposto para o PRRG

ximados, é proposto um conjunto de regras compondo um teste de redução para o grafo associado ao PRRG. A meta básica deste teste é a de reduzir o tamanho dos dados de entrada de cada instância do PRRG. Nas simulações efetuadas para diferentes dimensões, observamos uma redução média de $40 \%$ a $60 \%$ no tamanho do grafo associado (veja as colunas 2 e 3 da Tabela 1). Este nível significativo de redução viabiliza a solução de instâncias maiores usando técnicas exatas, como por exemplo, utilizando a formulação aqui proposta, onde o número de restrições exigidas é polinomial em relação ao tamanho do problema. Os benefícios destas regras em relação aos métodos aproximados são latentes, uma vez que possibilitam um uso mais amplo até mesmo de heurísticas sofisticadas como as metaheurísticas, que passam a explorar um maior número de ótimos locais, aumentando com isso, a possibilidade de atingir um ótimo global. Os próximos passos deste trabalho deverão incluir a adaptação destas regras e da formulação aqui proposta para outras variantes do PCV, onde apenas um subconjunto de vértices é visitado. 


\begin{tabular}{|l|c|c|c|c|c|}
\hline Inst. & Grafo_O & Grafo_R & GRASP_SR & GRASP_R & Ref. \\
\hline 50a & $50 / 10 / 30 / 10$ & $34 / 10 / 13 / 11$ & 3654.861 & 2777.557 & $32 \%$ \\
\hline $50 \mathrm{~b}$ & $50 / 30 / 10 / 10$ & $30 / 30 / 0 / 0$ & 4913.604 & 3929.884 & $25 \%$ \\
\hline 50c & $50 / 20 / 20 / 10$ & $30 / 20 / 8 / 2$ & 4432.408 & 3784.730 & $17 \%$ \\
\hline 100a & $100 / 20 / 40 / 40$ & $57 / 20 / 16 / 21$ & 6208.851 & 3966.146 & $57 \%$ \\
\hline 100b & $100 / 40 / 20 / 40$ & $48 / 40 / 2 / 6$ & 7014.612 & 5412.270 & $30 \%$ \\
\hline 100c & $100 / 35 / 35 / 30$ & $51 / 35 / 7 / 9$ & 7318.550 & 5248.457 & $39 \%$ \\
\hline 200a & $200 / 60 / 100 / 40$ & $77 / 60 / 5 / 12$ & 14081.643 & 7564.522 & $86 \%$ \\
\hline 200b & $200 / 80 / 80 / 40$ & $87 / 80 / 1 / 6$ & 15592.429 & 9799.628 & $59 \%$ \\
\hline 200c & $200 / 100 / 60 / 40$ & $100 / 100 / 0 / 0$ & 18188.964 & 13063.451 & $39 \%$ \\
\hline 300a & $300 / 90 / 140 / 70$ & $90 / 90 / 0 / 0$ & 21653.638 & 11351.215 & $91 \%$ \\
\hline 300b & $300 / 100 / 100 / 100$ & $117 / 100 / 4 / 13$ & 21227.462 & 12977.302 & $64 \%$ \\
\hline 300c & $300 / 140 / 90 / 70$ & $140 / 140 / 0 / 0$ & 25430.852 & 18778.253 & $35 \%$ \\
\hline
\end{tabular}

Tabela 1: Resultados Médios Comparativos das regras de redução e do algoritmo GRASP/VNS (Inst. = Instância; Grafo_O = Grafo Original; Grafo_R = Grafo Reduzido; GRASP_SR = Solução média usando Grafo_O; GRASP_R = Solução média usando Grafo_R; Ref. = Percentual de melhora com o uso das regras)

\begin{abstract}
The Covering Tour Problem is a job sequencing problem and it is defined on a graph $G=(V \cup W, E)$, where $W$ is a set of vertices that must be covered. The problem consists of determining a minimum length Hamiltonian cycle on a subset of $V$ such that every vertex of $W$ is within a given non negative distance $d$ from at least one node in the cycle. Being a generalization of the Traveling Salesman Problem, this problem is NP-Hard. This work presents a new mathematical formulation based on flow variables, reduction rules for the associated graph and an original metaheuristic algorithm to solve the Covering Tour Problem approximately.
\end{abstract}

\title{
Referências
}

[1] J. Current, Multiobjective Design of "Transportation Networks", Ph.D. Thesis, Department of Geography and Environmental Engineering, The Johns Hopkins University, 1981.

[2] T. Feo e M. Resende, Greedy randomized adaptive search procedure, Journal of Global Optimization 6 (1995), 109-133.

[3] M. Gendreau, G. Laporte e F. Semet, The covering tour problem, Operations Research 45 (1995), 568-576.

[4] V. Maniezzo, R. Baldacci, M. Boschetti e M. Zamboni, "Scatter Search Methods for The Covering Tour Problem", Scienze dell'Informazione, University of Bologna, 1999.

[5] N. Mladenovic e P. Hansen, Variable neighborhood search, Computers and Operations Research 24 (1997), 1097-1100. 
[6] G. Laporte, A. Asef-Vaziri e C. Sriskandarajah, Some applications of the generalized Travelling Salesman problem, J. Oper. Research Society 47 (1995), 1461-1467. 